\title{
Tuberculosis case notification data in Viet Nam, 2007 to 2012
}

\author{
Nguyen Viet Nhung, ${ }^{a}$ Nguyen Binh Hoa, ab Pham Huyen Khanhc and Cornelia Hennig ${ }^{c}$ \\ Correspondence to Nguyen Bin Hoa (e-mail: nguyenbinhoatb@yahoo.com).
}

Tuberculosis (TB) remains a major cause of morbidity and mortality, and Viet Nam ranks 12 among the 22 high-TB burden countries. This study analyses surveillance data of the National Tuberculosis Control Programme in Viet Nam for the six-year period 2007 to 2012. During the study period, 598877 TB cases (all forms) were notified, and 313225 (52.3\%) were new smear-positive cases. The case notification rate of new smear-positive cases was decreased, from 65 per 100000 population in 2007 to 57 per 100000 population in 2012; this decrease was observed for males and females in all age groups except males aged 0-14 and females aged 15-24 years. The male-to-female ratio of new smear-positive TB cases increased from 2.85 in 2007 to 3.02 in 2012 . The average annual cure rate of new smear-positive cases was $90.3 \%$. The high male-to-female ratio for new smear-positive TB cases in this notification data was lower than that from the 2007 TB prevalence survey in Viet Nam, suggesting a lower case detection for males. The decrease in new smear-positive case notification rates may reflect a decline in TB incidence in Viet Nam as several programmatic improvements have been made, although further research is required to increase case detection among young males and children.

$\mathrm{V}$ iet Nam has approximately 90 million inhabitants in 63 provinces, 700 districts and 11145 communes. ${ }^{1}$ Tuberculosis (TB) remains a major cause of morbidity and mortality, with Viet Nam ranking 12 among the 22 high-TB burden countries. ${ }^{2}$ In 2012, the estimated TB incidence (including HIVpositive patients) was 147 per 100000 population; prevalence was 218 per 100000 population; and mortality (excluding HIV) was 20 per 100000 population. TB incidence among HIV-positive cases was estimated to be 10 per 100000 population. $^{2}$

The Viet Nam National Tuberculosis Control Programme (NTP) commenced in 1986, with the directly observed treatment, short-course (DOTS) strategy initiated in 1992, reaching $100 \%$ DOTS coverage by $2000 .{ }^{3}$ The Viet Nam TB Information Management Electronic System (VITIMES) was established in 2009 and is a web-based system designed to collect patientbased data on patients screened for TB, on notified TB cases and on the treatment outcomes of TB patients. Data sources comprise paper reports, existing registers and laboratory forms. VITIMES was introduced in two phases: Phase I at the provincial level with aggregated data being entered from quarterly district paper reports and Phase II at the district level where individual patient information is entered. Phase I was implemented by all
63 provinces in 2010; Phase II coverage is expected nationwide by 2015 .

The objectives of this report are to assess case notification rates of all TB and new smear-positive TB cases over time and to provide a summary by age, sex and treatment outcomes in Viet Nam from 2007 to 2012.

\section{METHODS}

This descriptive study used TB surveillance data routinely collected by NTP from 2007 to 2012, disaggregated by age and sex, as well as treatment outcome reports from aggregate district-level reports entered into VTIMES.

Data were exported to EpiData and analysed using Excel and EpiData (Version 2.2, http://www.epidata. $\mathrm{dk}$ ). Differences in proportions were assessed using the chi-square test, and differences in case notification rates were assessed using poison regression testing. Point estimates are shown with 95\% confidence intervals (Cl) for proportions.

The TB patient categories followed those described in the Revised international definitions in tuberculosis control, ${ }^{4}$ and the positivity rate of presumptive TB cases

\footnotetext{
a National Tuberculosis Programme Vietnam, Ha Noi, Viet Nam.

b Centre for Operational Research, International Union Against Tuberculosis and Lung Disease, Paris, France.

Office of the WHO Representative in Viet Nam, Ha Noi, Viet Nam. Submitted: 27 April 2014; Published: 9 February 2015

doi: 10.5365/wpsar.2014.5.2.005
} 
Figure 1. Number of presumptive TB patients examined by sputum microscopy; number of sputum smear-positive cases detected and positivity rate, ${ }^{*}$ Viet Nam, 2007-2012

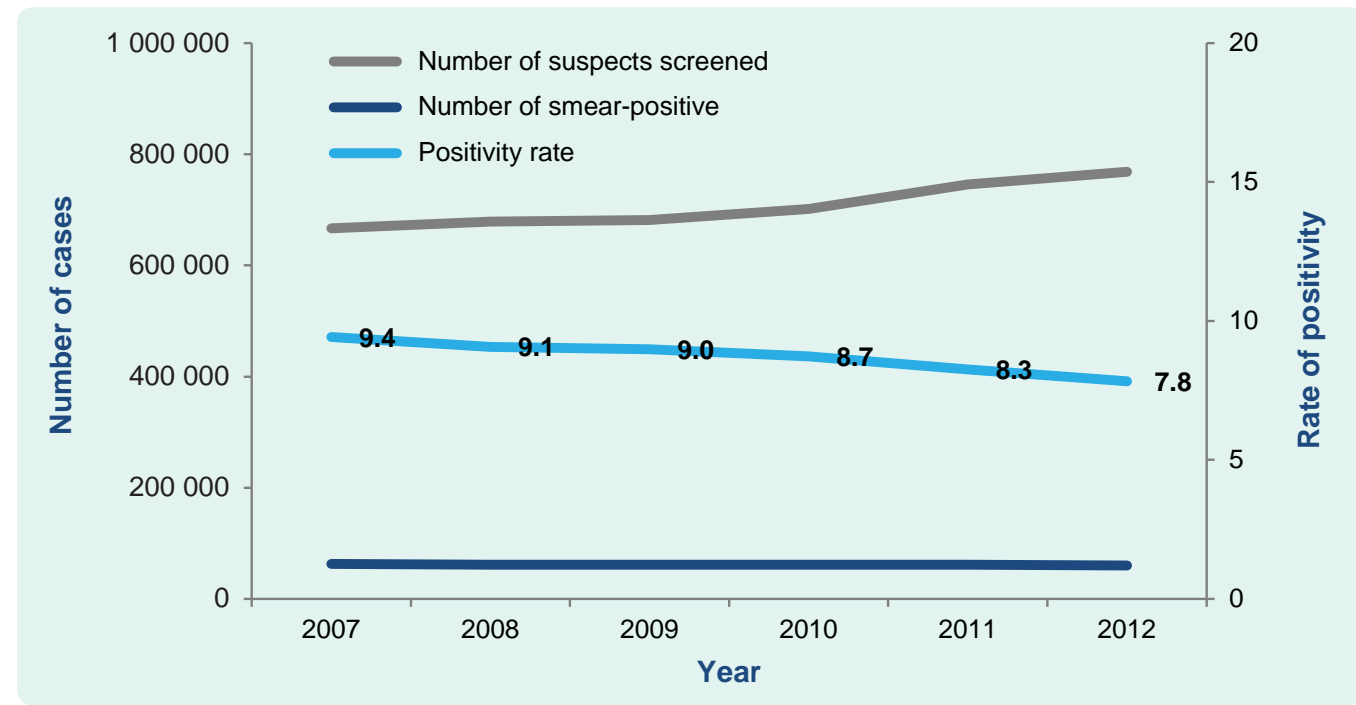

* Positivity rate: the number of sputum smear-positive cases divided by the total number of presumptive tuberculosis cases screened by microscopy.

was the number of sputum-positive cases divided by the total number of presumptive TB cases screened by microscopy.

\section{RESULTS}

\section{TB cases}

Between 2007 and 2012, a total of 4243630 presumptive TB cases were examined by sputum smear microscopy, with 368594 (8.7\%) being smear-positive. The number screened increased from 666947 in 2007 to 768449 in 2012, with the positivity rate significantly decreased from $9.4 \%$ in 2007 to $7.8 \%$ in $2012(P<0.001$, Figure 1$)$.

There were 598877 TB cases (all forms) notified: 313225 (52.3\%) were new smear-positive, 120769 (20.2\%) were smear-negative, 115450 (19.3\%) were extrapulmonary, and 49433 (8.3\%) were cases previously treated. The proportion of new smear-positive cases decreased from $55.4 \%$ in 2007 to $49.1 \%$ in $2012(P<0.001)$; in contrast, the proportion of previously treated cases increased from $7.8 \%$ in 2007 to $8.7 \%$ in $2012(P<0.001)$, and the proportion of new smear-negative cases increased from $17.8 \%$ in 2007 to $22.4 \%$ in $2012(P<0.001$, Figure 2$)$. While the annual case notification rate of TB (all forms) remained stable over the study period (range 114 to 117 per
100000 population, $P=0.749$ ), the case notification rate of new smear-positive TB cases significantly declined, from 65 to 57 per 100000 population between 2007 and 2012, respectively $(P<0.001$, Figure 3$)$.

\section{New smear-positive cases}

The average annual case notification rate of new smear-positive TB cases for the period 2007 to 2012 was 60.4 per 100000 population (95\% Cl: 60.2-60.6). Age-specific case notification rates increased with increasing age group, from 0.5 per 100000 population ( $95 \% \mathrm{Cl}: 0.5-0.6$ ) for the $0-14$ year age group to 162.9 per 100000 population (95\% Cl: 161.6-164.2) for those aged 65 years and above (Table 1).

There was a significant decline in the smear-positive notification rate between 2007 and 2012 for all age groups ( $p<0.05$ for all). Sex-specific average annual case notification rates also declined between 2007 to 2012 for both males and females in each of the five age groups except for males aged $0-14$ and females aged 15-24 ( $P=0.324$ and $P=0.353$, respectively). In contrast, there was a significant increase in the smear-positive notification rate of female aged $0-14$ $(P<0.001$, Figure 4).

The total male-to-female ratio of new smear-positive TB cases increased from 2.85 in 2007 to 3.02 in 2012. 
Figure 2. Proportion of TB cases (all forms) by case classification and year, Viet Nam, 2007-2012

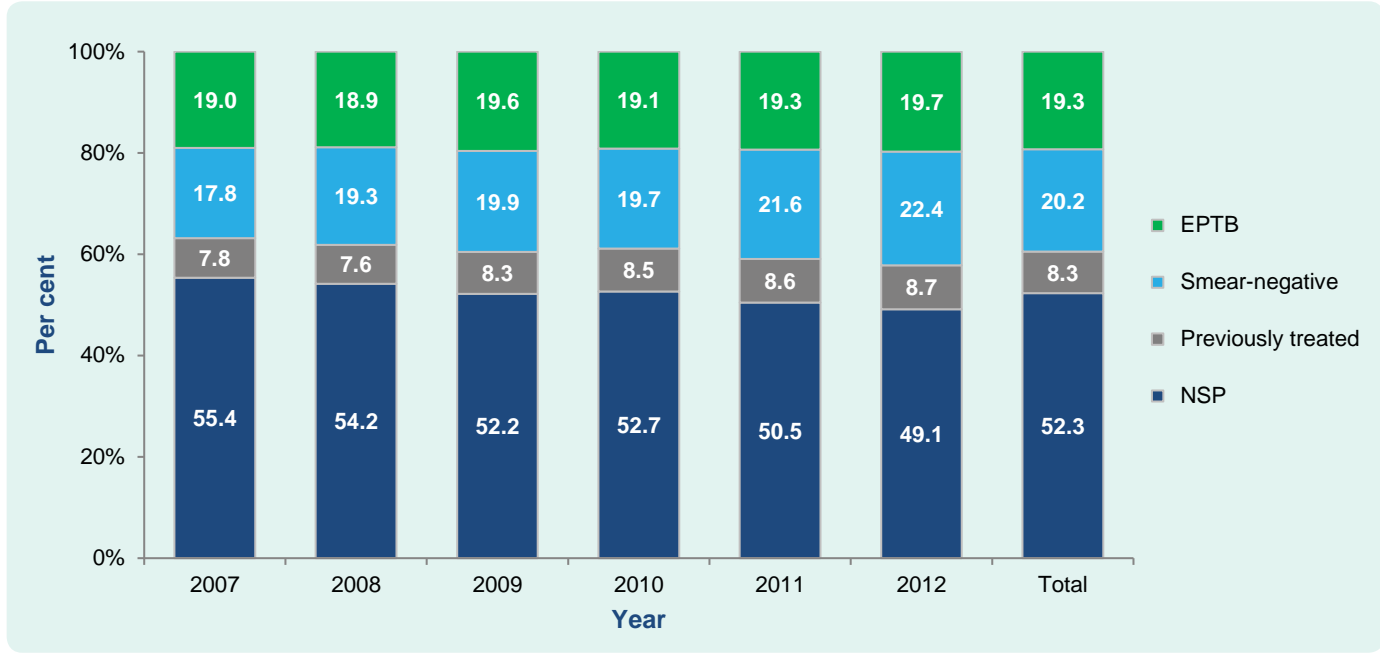

EPTB, extrapulmonary tuberculosis; NSP, new smear-positive tuberculosis.

Figure 3. Case notification rate of TB (all forms) and new smear-positive TB cases per 100000 population, Viet Nam, 2007-2012

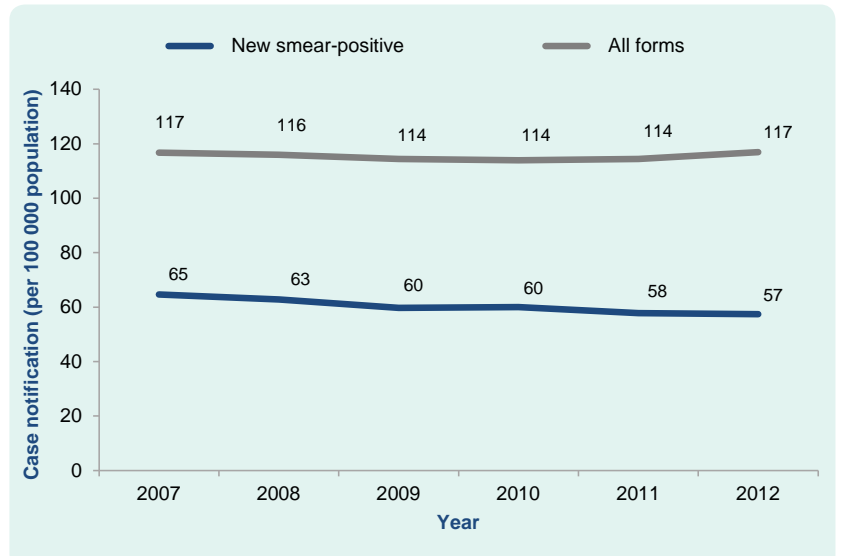

An observation: over the study period, age group-specific male-to-female ratios seemed to increase for the four age groups 35-44, 45-54, 55-64 and 65 year and above age groups, otherwise this ratios seemed to be constant or decreasing (Table 2).

The 2012 case notification rate of new smear-positive TB by province showed that rates are generally higher in the southern provinces compared with the northern provinces (Figure 5).

Although the number of children with smear-positive TB increased from 84 in 2008 to 142 cases in 2012, the proportion of children aged 0-14 among new smear-positive TB cases was low (0.2\%) during the period 2007 to 2012.

Table 1. Average annual case notification rate of new smear-positive TB cases by sex and age group, Viet Nam, 2007-2012

\begin{tabular}{|c|c|c|c|c|c|c|c|c|c|}
\hline \multirow{3}{*}{$\begin{array}{l}\begin{array}{c}\text { Age group } \\
\text { (years) }\end{array} \\
0-14\end{array}$} & \multicolumn{3}{|c|}{ Total } & \multicolumn{3}{|c|}{ Male } & \multicolumn{3}{|c|}{ Female } \\
\hline & \multirow{2}{*}{$\begin{array}{c}\begin{array}{c}\text { Notification } \\
\text { per } 100000 \\
\text { population }\end{array} \\
0.5\end{array}$} & \multicolumn{2}{|c|}{$\begin{array}{l}95 \% \text { confidence } \\
\text { interval }\end{array}$} & \multirow{2}{*}{$\begin{array}{c}\begin{array}{c}\text { Notification } \\
\text { per } 100000 \\
\text { population }\end{array} \\
0.5\end{array}$} & \multicolumn{2}{|c|}{$\begin{array}{l}\text { 95\% confidence } \\
\text { interval }\end{array}$} & \multirow{2}{*}{$\begin{array}{c}\begin{array}{c}\text { Notification } \\
\text { per } 100000 \\
\text { population }\end{array} \\
0.6\end{array}$} & \multicolumn{2}{|c|}{$\begin{array}{l}95 \% \text { confidence } \\
\text { interval }\end{array}$} \\
\hline & & 0.5 & 0.6 & & 0.4 & 0.5 & & 0.5 & 0.6 \\
\hline $15-24$ & 32.4 & 32.1 & 32.8 & 40.2 & 39.7 & 40.8 & 24.4 & 23.9 & 24.8 \\
\hline $25-34$ & 67.3 & 66.8 & 67.9 & 101.2 & 100.2 & 102.2 & 34.0 & 33.5 & 34.6 \\
\hline $35-44$ & 75.0 & 74.4 & 75.6 & 123.6 & 122.5 & 124.7 & 26.8 & 26.2 & 27.3 \\
\hline $45-54$ & 97.6 & 96.9 & 98.4 & 165.0 & 163.6 & 166.5 & 34.9 & 34.2 & 35.5 \\
\hline $55-64$ & 133.7 & 132.5 & 134.9 & 218.9 & 216.6 & 221.3 & 61.4 & 60.3 & 62.6 \\
\hline 65 and above & 162.9 & 161.6 & 164.2 & 260.3 & 257.7 & 263.0 & 97.9 & 96.6 & 99.3 \\
\hline Total & 60.4 & 60.2 & 60.6 & 90.7 & 90.3 & 91.1 & 30.8 & 30.5 & 31.0 \\
\hline
\end{tabular}


Figure 4. Case notification rate of new smear-positive TB cases by sex, age group and year, Viet Nam, 2007-2012*
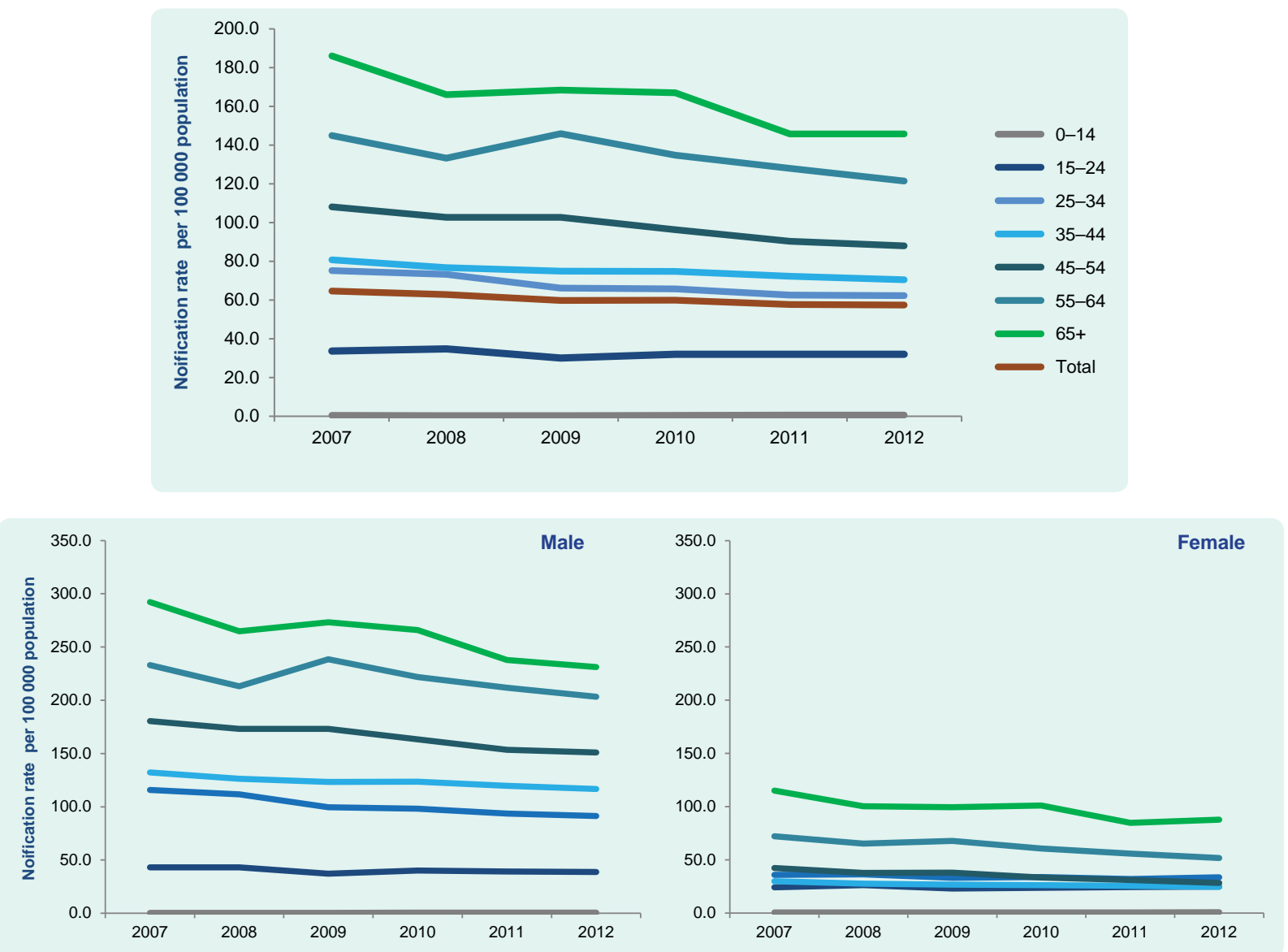

* $P$-value for all age groups was $<0.05$, except for the male $0-14$ age group $(p=0.324)$ and the female $15-24$ age group $(p=0.353)$.

Treatment outcome of new smear-positive cases

The cure rate of new smear-positive cases was maintained at high levels (above 85\%) for all years in the study period; although this increased slightly from $89.8 \%$ in 2007 to $91.1 \%$ in 2012 , the difference was not statistically significant $(P=0.089$, Figure 6$)$. The average annual cure rate for six years was $90.3 \%$. The six-year annual averages for the other treatment outcomes were: treatment completed at $2.0 \%$, death at $3.0 \%$, treatment failure at $1.1 \%$, treatment after default at $1.7 \%$ and transferred out at $1.9 \%$.

Table 2. Male-to-female ratio of new smear-positive TB cases by age group and year, Viet Nam, 2007-2012

\begin{tabular}{cccccccc}
\hline Age group (years) & $\mathbf{2 0 0 7}$ & $\mathbf{2 0 0 8}$ & $\mathbf{2 0 0 9}$ & $\mathbf{2 0 1 0}$ & $\mathbf{2 0 1 1}$ & $\mathbf{2 0 1 2}$ & Total \\
\hline $0-14$ & 0.8 & 0.7 & 0.8 & 1.0 & 0.9 & 0.6 & 0.8 \\
$15-24$ & 1.8 & 1.6 & 1.6 & 1.7 & 1.6 & 1.6 & 1.6 \\
$25-34$ & 3.2 & 3.1 & 3.0 & 2.9 & 2.9 & 2.7 & 3.0 \\
$35-44$ & 4.4 & 4.6 & 4.6 & 4.7 & 4.7 & 4.8 & 4.6 \\
$45-54$ & 4.3 & 4.6 & 4.6 & 4.9 & 5.0 & 5.3 & 4.7 \\
$55-64$ & 3.2 & 3.3 & 3.5 & 3.7 & 3.8 & 3.9 & 3.6 \\
65 and above & 2.5 & 2.6 & 2.7 & 2.6 & 2.8 & 2.6 & 2.7 \\
Total & 2.9 & 2.9 & 2.9 & 3.0 & 3.1 & 3.0 & 3.0 \\
\hline
\end{tabular}


Figure 5. Case notification rates of new smear-positive TB cases by province, Viet Nam, 2012

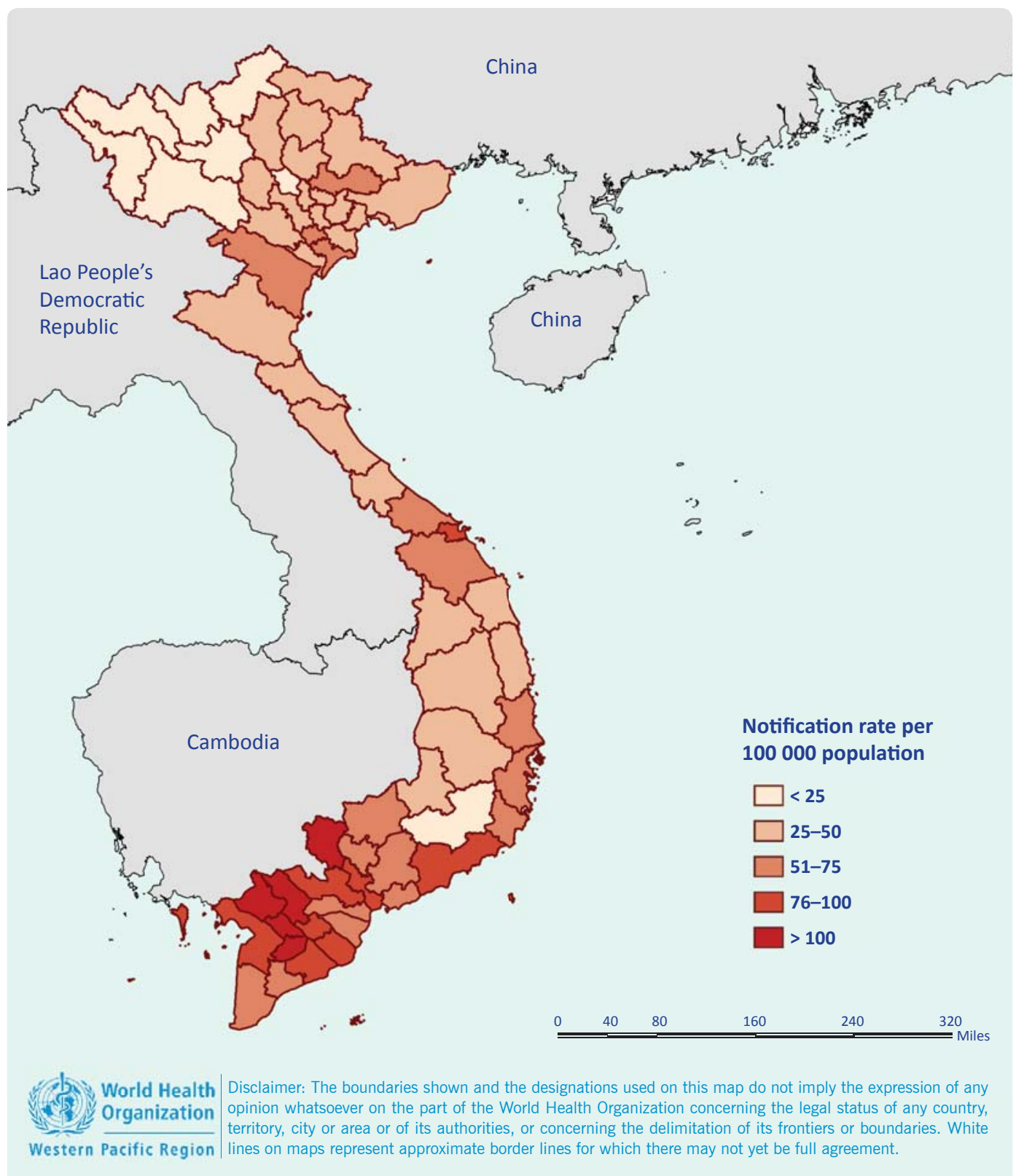

\section{DISCUSSION}

The data from the NTP in Viet Nam showed a stable case notification rate of all forms of TB for the years 2007 to 2012, ranging from 114 to 117 per 100000 population, yet a significant decrease for new smear-positive TB cases, dropping from 65 to 57 per 100000 population from 2007 to 2012, respectively. Whether this decrease in the new smear-positive case notification rate reflects an actual decrease in infectious cases of TB is an important programmatic question. The number of presumptive TB cases who had a sputum smear examination increased over the study period, with the positivity rate significantly decreasing from $9.4 \%$ to $7.8 \%$. During the same period the Viet Nam NTP focused on improving TB case finding by engaging private and public facilities outside the NTP; improving chest X-ray (CXR) availability and quality; introducing activities to reach presumptive TB cases in remote areas; and through advocacy, communication and social mobilization activities. The Viet Nam NTP also expanded TB activities in prisons, with the number of TB units in prisons expanding from 24 in 2007 to 51 in 2012. In addition, the quality of sputum examination has improved each year and the proportion of HIV coinfection among TB patients was reduced from 8\% 
Figure 6. Proportion of new smear-positive TB cases by treatment outcome and year, Viet Nam, 2007-2012

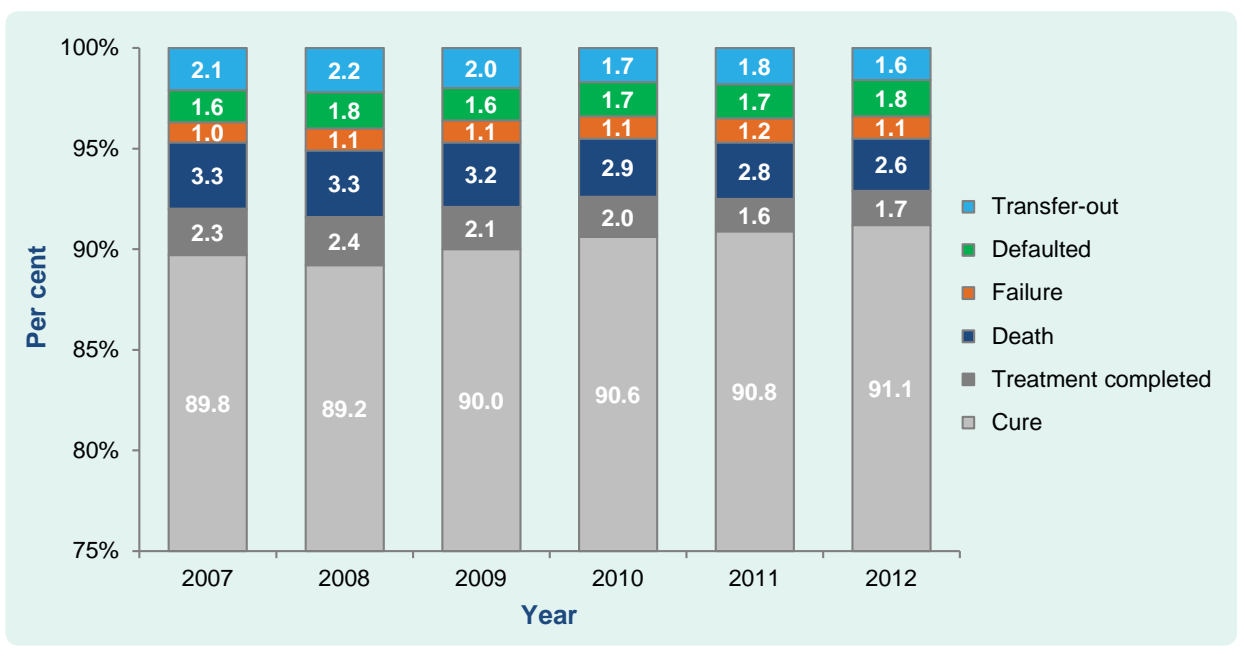

in 2007 to $7 \%$ in $2012 .^{2}$ Case reporting has also been improved by introducing electronic reporting systems. The latest World Health Organization (WHO) estimates for TB (January 2013) also suggest that the burden of TB in Viet Nam is estimated to decline in incidence by $2.6 \%$ per year, and in prevalence and mortality by $4 \%$ and $5 \%$ per year, respectively. ${ }^{5}$

In our study, the smear-positive TB case notification rates in Viet Nam increased with age; this trend has also been reported in many other countries., The first national tuberculosis prevalence survey in Viet Nam (2006-2007) also showed a strong and statistically significant correlation between increasing age and the prevalence of CXR abnormalities, persistent productive cough and bacteriologically confirmed TB. ${ }^{6}$ In our study, more than half of new smear-positive cases notified in 2012 (54\%) were aged 45 years and above. This is in contrast to the proportion of persons in the population aged 45 years and above, which was only $28 \% .^{7}$

Demographic changes in population over time may affect trends in TB notification rates. ${ }^{8}$ Viet Nam population data have shown an increase in the proportion of persons aged 45 and above from 25\% in 2007 to $28 \%$ in $2012 .{ }^{7}$ This increase in the older population may cause a lower-than-expected decline in the overall case notification rates of TB as older people have higher TB notification rates, and younger persons have lower TB notification rates but make up a smaller proportion of the population.
To control for this demographic factor, we also analysed the notification rates of new smear-positive TB by age group over time. There was a significant decline in the smear-positive notification rate between 2007 and 2012 for all age groups except for males aged 0-14 years and females aged 15-24 years. This finding differs from that observed for notified cases in the period 1997 to 2004, when TB notification decreased in all age groups except among young men in the $15-35$ year age group. ${ }^{9}$

In this study, the proportion of children among new smear-positive TB cases was less than the estimated proportion of TB in children in low-income countries. ${ }^{10}$ This low proportion may be due to there being less smear-positive TB occurring in children or because of underreporting of childhood TB to the NTP. As a result of this, since 2012, the NTP has been intensifying efforts to diagnose and notify childhood TB by strengthening TB case finding and screening of children in close contact with an adult TB case.

We observed that the male-to-female ratio in notified smear-positive cases increased from 2.85:1 in 2007 to $3.02: 1$ in 2012 . This ratio is much higher than the male-to-female ratio among notified new smear-positive cases in the 22 high-burden countries (1.9:1), in the WHO Western Pacific Region (2.4:1) and worldwide $(1.9: 1){ }^{2}$ The 2006 to 2007 national TB prevalence survey in Viet Nam reported a maleto-female ratio of $4.8: 1$ for new smear-positive cases and 4.6:1 in bacteriologically confirmed $\mathrm{TB}^{6}{ }^{6}$ These 
data support the hypothesis that the difference in TB notification rates in males and females in Viet $\mathrm{Nam}$ reflects a true difference in disease occurrence rather than a difference in access to diagnosis and treatment as previous studies have suggested. ${ }^{11}$ The higher maleto-female ratio in the prevalence survey compared to the notification data suggests lower case detection in males compared with females. A possible explanation for this preponderance of males might be the large difference in the proportion of men and women who smoke. ${ }^{12}$ In 2008, WHO reported that in Viet Nam, $2 \%$ of women are current smokers compared to $44 \%$ of men, and $34 \%$ of men smoke daily. ${ }^{13}$ Other possible explanations for the high male-to-female ratio may be the higher HIV prevalence among males compared to females ${ }^{14}$ and higher alcohol consumption among men. ${ }^{15}$ More research to investigate potential differences in the epidemiology of TB between males and females is needed as well as improved case finding among men.

This analysis was based on notification data reported to VITIMES. For the results to truly reflect the TB epidemiology in Viet Nam, they are dependent on not only the quality of the surveillance system but also the capacity of the NTP to detect and treat all TB cases in the community. The TB surveillance system in Viet Nam has been assessed as a well-functioning system with many strengths but also several gaps. ${ }^{5}$ First, the Viet Nam NTP does not yet collect information on sex and age for all TB cases; this is currently only available for new smear-positive TB cases. Second, not all care providers in the public and private sectors report to the NTP, resulting in notification gaps, including for childhood TB, which suggests an underreporting of cases. Third, as VITIMES is not yet implemented in all districts providing individual case-based data, our data analysis was based on aggregated reports from district levels which restrict analysis. The TB information system should be further strengthened to improve data quality and coverage in non-NTP facilities.

Despite these limitations, this report has shown an increase in TB testing in Viet Nam coupled with a decrease in notification of new smear-positive cases between 2007 and 2012. The case notification rate of smear-positive TB increased significantly with age, and a male-to-female ratio lower than the 2007 prevalence survey suggests lower case detection in males compared with females. These results suggest that the NTP activities to increase case detection have been effective, although further research is required to increase case detection among young males and children.

\section{Conflicts of interest}

None declared.

\section{Funding}

None.

\section{Acknowledgements}

The authors would like to thank the staff of the Viet Nam National Tuberculosis Control Programme, especially the data management team.

\section{References}

1. General Statistics Office. Ha Noi, Government of Viet Nam, 2014 (http://www.gso.gov.vn/default.aspx?tabid=386\&idmid=3\&Item $I D=14154$, accessed 18 December 2014).

2. Global tuberculosis report 2013. Geneva, World Health Organization, $2013 \quad$ (http://apps.who.int/iris/ bitstream/10665/91355/1/9789241564656_eng.pdf, accessed 18 December 2014).

3. Huong NT et al. Establishment and development of the National Tuberculosis Control Programme in Viet Nam. International Journal of Tuberculosis and Lung Disease, 2005, 9:151-156. pmid: 15732733

4. World Health Organization, International Union Against Tuberculosis and Lung Disease, Royal Netherlands Tuberculosis Association. Revised international definitions in tuberculosis control. International Journal of Tuberculosis and Lung Disease, 2001, 5:213-215. pmid:11326818

5. Bloss $\mathrm{E}$ et al. Evaluation of TB surveillance in Viet Nam. Mission report, 14-18 January 2013.

6. Hoa NB et al. National survey of tuberculosis prevalence in Viet Nam. Bulletin of the World Health Organization, 2010, 88:273-280. doi:10.2471/BLT.09.067801 pmid: 20431791

7. Population pyramid, Viet Nam 2012. Belgium, Cooperation Exchange Training, 2014 (http://www.coopami.org/en/countries/ other_countries/vietnam/country_description/index.htm, accessed 18 Décember 2014).

8. Hiatt T, Nishikiori N. Epidemiology and control of tuberculosis in the Western Pacific Region: analysis of 2012 case notification data. Western Pacific Surveillance and Response Journal, 2014, 5:2534. doi:10.5365/wpsar.2014.5.1.013 pmid:24734214

9. Vree $\mathrm{M}$ et al. Tuberculosis trends, Vietnam. Emerging Infectious Diseases, 2007, 13:796-797. doi:10.3201/eid1305.060904 pmid: 18044050

10. Marais BJ et al. The burden of childhood tuberculosis and the accuracy of community-based surveillance data. International Journal of Tuberculosis and Lung Disease, 2006, 10:259-263. pmid: 16562704

11. Rieder HL. Epidemiologic basis of tuberculosis control. Paris, International Union Against Tuberculosis and Lung Disease, 1999. 
12. Holmes CB, Hausler H, Nunn P. A review of sex differences in the epidemiology of tuberculosis. International Journal of Tuberculosis and Lung Disease, 1998, 2:96-104. pmid:9562118

13. WHO report on the global tobacco epidemic, 2008. Geneva, World Health Organization, 2008 (http://www.who. int/tobacco/mpower/mpower_report_full_2008.pdf, 18 December 2014).
14. Thanh $\mathrm{DH}$ et al. HIV infection among tuberculosis patients in Viet Nam: prevalence and impact on tuberculosis notification rates. International Journal of Tuberculosis and Lung Disease, 2010, 14:986-993. pmid:20626943

15. Global status report on alcohol and health 2011. Geneva, World Health Organization, 2011 (http://www.who.int/substance_abuse/ publications/global alcohol report/msbgsruprofiles.pdf, accessed

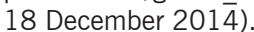

